\title{
INVESTIGATION OF NEAR SURFACE DISTORTIONS IN Si SINGLE CRYSTALS BY MEANS OF SPATIAL DISTRIBUTION ANALYSIS OF REFLECTED BEAMS
}

\author{
V.I. Khrupa, S.M. Krasulya, V. Machulin, L.I. Datsenko \\ Institute of Semiconductor Physics, National Academy of Sciences \\ Pr. Nauki, 252028 Kiev, Ukraine
}

and J. AUleytner.

Institute of Physics, Polish Academy of Sciences

Al. Lotników 32/46, 02-668 Warszawa, Poland

\begin{abstract}
A diffractometrical method for quantitative evaluation of structure perfection level in silicon single crystals containing various types of near surface distortions is described. The method is based on the spatial distribution analysis of the reflected intensity in the Bragg case of diffraction. To implement the proposed approach one has to satisfy the condition of the so-called low $\mathrm{X}$-ray absorption because in this case the penetration depth of diffracted radiation exceeds the corresponding value of extinction length. It permits us to obtain a remarkable value of noncoherent reflectivity due to defects placed in deep (on the extension of absorption length) regions of a crystal and therefore, to increase the sensitivity of scattering for low distortions of crystal lattice. Using the method described here the extension of various disturbed layers as well as the level of the static Debye-Waller factor of a crystal can be determined. The effect of surface distortions caused by mechanical treatment and the influence of the following thermal annealing as well as irradiation by high energy protons on the defective structure of the samples were investigated.
\end{abstract}

PACS numbers: 81.40.-z, 61.10.-i

$\mathrm{X}$-ray diffractometry based on determination of the integral characteristics (i.e. averaged over a scattering volume) of defect structures is one of the most effective methods for diagnostics of real single crystals. Now the nondestructive methods of rapid structure perfection control are developed and widely used in scientific and industrial laboratories. The methods, in particular, permit us to identify the type of predominate defects distributed homogeneously over the bulk of a crystal as well as to determine their mean dimension and concentration $[1,2]$. 
Similar approaches based on the analysis of the diffraction peak position, the shape of rocking curve as well as the reflectivity of a crystal, are applicable, in some cases, for determination of structure characteristics of damaged near surface layers (DSL) in rather perfect crystals.

Structure diagnostics of crystals containing simultaneously surface and volume distortions of a lattice are believed to be rather a difficult problem because the DSL and defects situated in the bulk of a crystal affect the diffraction parameters in a similar way. To solve this task, we have investigated the regularities of spatial distribution of X-ray intensity reflected in Bragg geometry of diffraction by real crystals containing both kinds of the above-mentioned imperfections. On the base of the obtained data the method of rapid nondestructive diagnostics of technologically distorted surface and volume defects was developed.

Surface distortions were created by means of either abrasive grinding or irradiation of silicon crystals with high energy $(E=6 \mathrm{MeV})$ protons. The following samples, prepared in the shape of plane-parallel plates, were investigated in our experiments. The sample 1 grown by floating zone method was studied in as-grown state. The sample 2 obtained by Czochralski method was annealed at $850^{\circ} \mathrm{C}$ for 4 hours to create microdefects in bulk. According to X-ray topography these defects have a cluster nature with radii up to $10 \mu \mathrm{m}$. The sample 3 grown also by Czochralski method was irradiated by protons at the accelerator U-120 (Kiev, Ukraine).

Surfaces of the samples 1 and 2 (crystallographic planes $\{111\}$ were ground on both sides of plates by a free abrasive with a particle diameter $(M=20 \pm$ $5 \mu \mathrm{m})$ to prevent crystals from macroscopic elastic bending [3]. The thickness $t=1.4 \mathrm{~mm}$ of plates was chosen from the condition: $2 t_{\mathrm{d}} \ll t<\mu^{-1}$, where $t_{\mathrm{d}}$ is the approximate thickness of the DSL, $t_{\mathrm{d}} \cong 3 M ; \mu^{-1}$ is the absorption length of applied X-rays.

Measurements of the spatial distribution of diffraction beam intensity $I(x)$ were performed by means of a single crystal spectrometer. The characteristic $\operatorname{Ag} K_{\alpha_{1}}$ radiation $\left(\mu=7.4 \mathrm{~cm}^{-1}\right)$ was used. The scheme of diffraction pattern formation is given in Fig. 1a. The thin ribbon beam of X-rays collimated by the slits $S_{1}=S_{2}=0.05 \mathrm{~mm}$ falls on the entrance recorded by the detector D with the narrow slit $S_{3}\left(S_{3} \leq S_{1}, S_{2}\right)$ along the direction perpendicular to the reflected beam.

Intensity of the reflected beam in the case of an imperfect crystal is known to consist of coherent, $I_{\mathrm{B}}$, and diffuse, $I_{\mathrm{D}}$, components, describing the diffraction on an averaged lattice and on defects, respectively. According to Ref. [4] $I_{\mathrm{B}}$ forms the main peak with a maximum at $x=0(x$ is the distance from the point of initial beam incidence on the surface of a crystal). This component diminishes considerably in the depth approximately of the extinction length $A$. As concerns $I_{\mathrm{D}}$, this component decreases noticeably at the absorption length $\mu^{-1}$ of radiation. So if the condition $A \ll \mu^{-1}$ takes place, the intensity measured at $x>A$ consists mainly of the diffuse term which depends on the level of structure distortions as well as on a character of defect distribution in the scattering volume [5].

The diagrams of reflected beam intensity distribution for the sample 1 are presented in Fig. 1b. They clearly show that the DSL due to mechanical treatment 


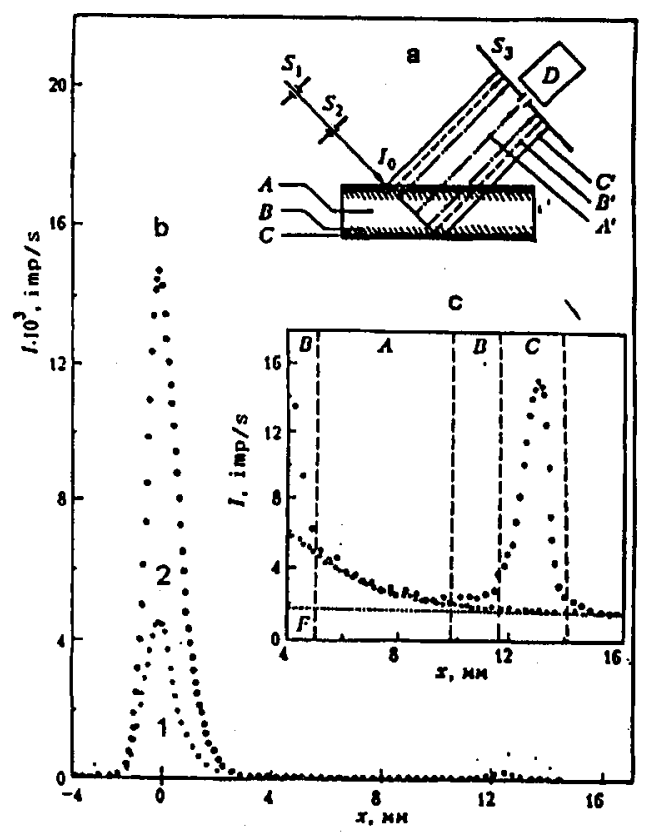

Fig. 1. The scheme illustrating appearing of diffraction image (a) and space diagrams of intensity distribution for reflex $I(x)$ (b); for crystal in the initial state of sample (1) and after surface polishing (2); periphery profile $I(x)$ in magnification (c). The sample 1 , reflex $333 ; \mathrm{A}$ - crystal matrix (bulk), $\mathrm{B}$ - area of elastic deformation, $\mathrm{C}$ - area of plastic deformation, $A^{\prime}, B^{\prime}, C^{\prime}$ - diffraction beams corresponding to these deformations, $\mathrm{F}$ - background, $\mathrm{D}$ - scintillator.

of the surfaces changed considerably the shape of the profile $I(x)$. First of all one can see an increase in the main peak intensity as well as peak width. These transformations are similar to those observed earlier [6] by excitation of model long wave ultrasonic vibrations in a perfect crystal. So, in the first approximation, the observed transformation of $I(x)$ can be explained by the effect of long range components of elastic displacement field caused by local surface concentrations of stresses. The fact that short annealing $\left(5 \mathrm{~min} ., 650^{\circ} \mathrm{C}\right)$ affects $I(x)$ testifies such relaxation of elastic strains in the crystalline matrix due to which one can observe an intensity decrease in the main peak and its width (Fig. 2A).

Besides, in Fig. 1 one can see appearance of an additional maximum coming from the zone $\mathrm{C}^{\mathrm{I}}$ (Fig. 1a) of crystals with the DSL due to X-ray scattering near the distorted exit surface. Annealing of the ground crystal changes the intensity of this maximum only there where its width decreases more distinctly (Fig. 2B). This fact permits us to suppose that the main cause of additional peak appearance is connected with incoherent X-ray scattering by defects in regions of plastically deformed near the surface layer. The elastic strain effect, being weaker in this case, is connected mainly with growing intensity at some distance from the second peak (zone $\mathrm{B}^{\mathrm{I}}$ in Fig. 1c). For the samples 1 and 2 one can determine the value of the 


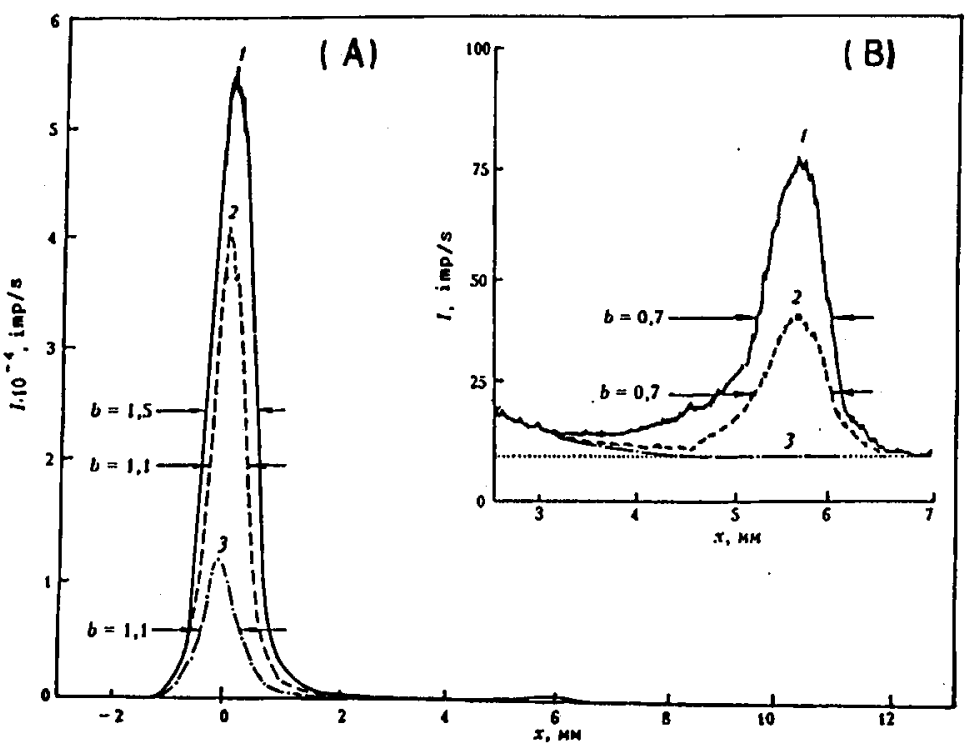

Fig. 2. The change of profile shape $I(x)$ during thermal treatment: (A) - the main peak, (B) - periphery profile. The sample 1, reflex 555, 1 - after polishing, 2 - after annealing for 5 minutes at the temperature of $650^{\circ} \mathrm{C}, 3$ - after chemical polishing; $b$ - half-width of peaks $(\mathrm{mm})$.

TABLE

Structural characteristics for the chemically polished sample 2 and that for mechanically treated surface (grinding).

\begin{tabular}{l|c|c|c|c|c|c}
\hline \hline Kind of treatment & $L \times 10^{2}$ & $\mu_{\mathrm{d}}\left(\mathrm{cm}^{-1}\right)$ & $r(\mu \mathrm{m})$ & $\begin{array}{c}n \times 10^{-4} \\
\left(\mathrm{~cm}^{-3}\right)\end{array}$ & $l_{\mathrm{p}}^{*}(\mu \mathrm{m})$ & $l_{\mathrm{e}}^{*}(\mu \mathrm{m})$ \\
\hline Chemical polishing & 1.7 & 0.8 & 2.2 & 3 & - & - \\
\hline Grinding & 1.8 & 0.9 & 2.3 & 3 & 65 & 120 \\
\hline
\end{tabular}

${ }^{*} l_{\mathrm{p}}$ and $l_{\mathrm{c}}$ are the length of plastic and elastic deformation zones, respectively.

distorted layers $B^{I}$ and $C^{I}$ extent as well as zones of the elastic strains. The results of these estimations are given in Table. They are close to the literature data.

It is of importance to note that the intensities in the central part of the diagram $I(x)$ coincide practically with initial as well as ground crystals (zone $\mathrm{A}^{\mathrm{I}}$ in Fig. 1b,c). This result testifies the main volume of samples (within the depth approximately from 0.45 to $0.99 \mathrm{~mm}$ from the entrance surface) being distorted by the elastic strains insignificantly. So, the diffracted beams are formed on this depth due to diffuse scattering of X-rays mainly on structural defects. The last circumstance may be used for determination of the integral characteristics of structural perfection, i.e. the static Debye-Waller factor $L$ as well as the coefficient of additional weakening of X-rays due to the diffuse scattering $\mu_{\mathrm{d}}$ on defects [3]. The dependences $L$ on the reflection order $H$ from the plane (111) 
$\left(|H|=\left(h^{2}+k^{2}+l^{2}\right)^{1 / 2}\right.$, where $h, k, l$ are the Miller indexes) have been plotted for determination of predominate distortions nature in the samples 2 and 3 using the experimental method [5].The obtained data testify the dependences $\ln L=f(\ln H)$ taken in initial state of both samples and after grinding correlate between them. The dependence $\ln L=f(\ln H)$ slope is close to 2 which corresponds to the theoretical dependence for small clusters [1].

The results concerning the $L$ and $\mu_{\mathrm{d}}$ parameters obtained by means of the $I(x)$ dependence analysis were used for calculation of the typical radii $r$ and concentration $n$ of microdefects in the sample 2. They are presented in Table and they are in agreement with the known literature data [2].

Concerning the sample 3 irradiated by high energy protons the developed method allowed us to discover a distorted zone where the slowed down hydrogen nuclei have stopped $(t \approx 1400 \mu \mathrm{m})$.

In conclusion it is necessary to note that the integral intensity distribution analysis of reflected radiation in the Bragg case of diffraction in crystals with combined distortions of structure (situated simultaneously in a bulk and near the surface) permits us to determine not only the depth of the DSL but also to obtain information about the type of predominant defects, their extensions and concentration.

This work was partially supported by the Ukrainian Committee for Science and Technology as well as by the Committee for Scientific Research (Poland), grant No. 2.PO31302109.

\section{References}

[1] L.I. Dátsenko, V.B. Molodkin, M.E. Osinovskii, Dynamicheskoe rasseyanie rentgenovskih luchey realnymi kristallami, Naukova dumka, Kiev 1988, p. 198 (in Russian).

[2] V.I. Khrupa, Metallophysika 13, 49 (1991).

[3] L.I. Datsenko, E.N. Kislovskii, Vestnik AN USSR 2, 57 (1989).

[4] E.N. Kislovskii, V.P. Kladiko, A.V. Fomin, V.I. Khrupa, Zavod. lab. 51, 30 (1985).

[5] I.V. Voskobojnikova, Neorganicheskie materiali 9, 1878 (1973).

[6] I.L. Shulpina, Ph. Thesis, Leningrad 1986, p. 37.

[7] I.R. Entin, V.I. Khrupa, J. Appl. Crystallogr. 24, 403 (1991). 